\title{
Microstructural Characterization of Porous Nanoceramic Composite Structures
}

\author{
Lauraine Denault, Anthony Y. Ku, Seth T. Taylor, and Sergio M. Loureiro
}

General Electric Global Research, 1 Research Circle, Niskayuna, NY, 12309

Mesoporous silica produced using surfactant-templated methods has attracted a great deal of interest for applications ranging from filtration to catalysis due to its organized, tunable, monodisperse, nanometer-sized porosity [1,2]. Well-defined porosity results from the encapsulation and subsequent removal of a self-assembling surfactant template inside a silica gel. Control over the pore size and organization is achieved through a variety of means, including the choice of surfactant, addition of ternary components to the precursor solution, and application of an external field $[3,4]$. It is possible to prepare mesoporous silica with single pore organization and pore size ranging from 2 to $30 \mathrm{~nm}$.

During process development for these types of structures, it is necessary to have a rapid screening tool that is capable of imaging the microstructure at a resolution of about $2 \mathrm{~nm}$ in poorly-conductive samples. Field emission SEM at low voltages has proven to be an excellent tool for this purpose. In this work, FESEM was used to image porous ceramic structures grown in porous anodic alumina templates. The local microstructures, particularly the organization of the porosity and the degree of filling, were studied.

The templates were typically as-received $25 \mathrm{~mm}$ diameter, 50 um thick Whatman anodic aluminum oxide (AAO) membranes with $200 \mathrm{~nm}$ pores. Porous ceramic structures inside the AAO pores were produced by immersing the membrane in a precursor solution, allowing evaporation-driven gelation, and subjecting the membrane to heat treatment. Challenges to FESEM analysis include the mechanical fragility of the material, its susceptibility to beam damage, and its tendency to charge. In addition these samples are hard to orient and the very fine porosity of the deposited material and variation in filling within the AAO pores further compound the charging problems. Samples were observed in both cross-section and plan view using a thin Pt coating to mitigate the charge and a beam energy of $5 \mathrm{keV}$.

Figure 1 shows typical SEM results. Figure $1(\mathrm{a}, \mathrm{b})$ shows P123-templated silica in AAO with a well-formed structures inside the AAO pores. However, these samples did not fill the entire volume of the AAO due to shrinkage during calcination. In cross-section (Fig. 1c), the shrunken regions retained the original shape of the AAO pore and remained attached to the AAO pore wall. Figure 1 (d) shows the structure obtained using F127 as the template which exhibit higher levels of filling. Sequential cross-sectional SEM images (Fig 1e) taken through the membrane showed good longitudinal filling and a cubic pore arrangement. TEM imaging has also been used to characterize these materials, and Fig. 2 shows a typical result from a P123/F127 templated pore. The TEM results will be discussed in more detail in the presentation.

SEM characterization is an important tool that allows for direct imaging and quick screening of these structures. When combined with other techniques such as TEM, XRD, BET surface area, gas permeability analysis, it provides detailed information about the local and bulk microstructure of the materials. 
1. C. T. Kresge, M. E. Leonowicz, W. J. Roth, J. C. Vartuli, J. S. Beck. Nature, 359, 710-712 (1992).

2. X. Feng et al., Science 276, 923 (1997).

3. J. C. Vartuli, et al. Chem. Mater. 6, 2317-2326 (1994).

4. I. A.. Aksay, et al. Science, 273, 892-898 (1996).
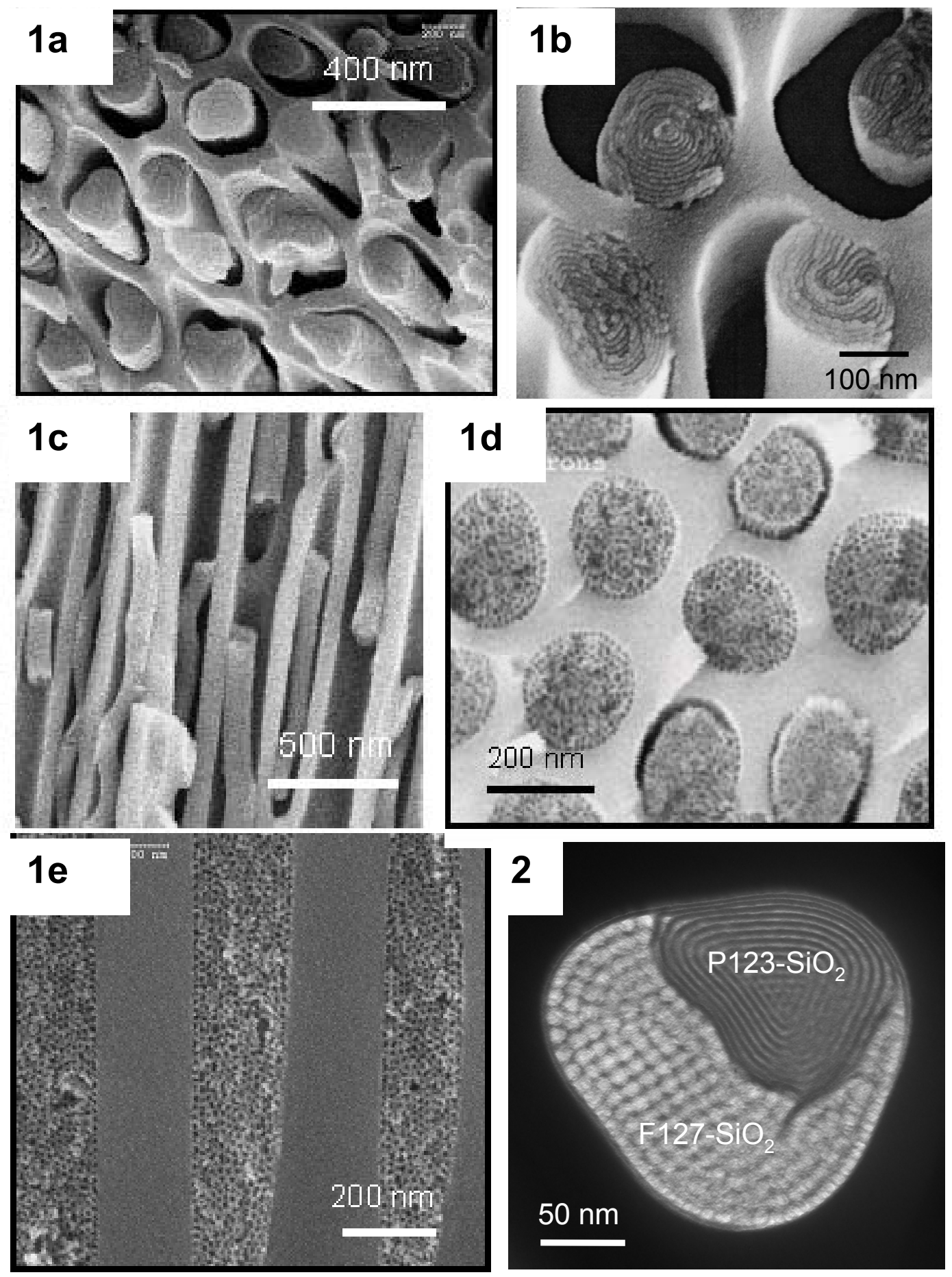

Figure 1. (a,b) Tilted view FESEM showing P123-templated silica in AAO (c) Cross-section of P123 silica. (d) Plan-view FESEM showing F127-templated silica in AAO. (e) Cross-section of F127 silica. Figure 2. TEM image of P123/F127 templated pore. 